\title{
Automatic Threshold Selection for BLM Signals during LHC Collimator Beam-Based Alignment
}

\author{
Gianluca Valentino* $^{* \dagger}$, Ralph W. Assmann ${ }^{\dagger \dagger}$, Roderik Bruce ${ }^{\dagger}$, Stefano Redaelli ${ }^{\dagger}$ and Nicholas Sammut ${ }^{* \dagger}$ \\ *University of Malta, Msida, Malta \\ ${ }^{\dagger}$ CERN, Geneva, Switzerland \\ $\ddagger$ DESY, Hamburg, Germany
}

\begin{abstract}
The Large Hadron Collider at CERN is the largest high-energy particle accelerator in the world. Proton beams are currently collided at an energy of $4 \mathrm{TeV}$ per beam to investigate the fundamental elements of matter. The collider is equipped with a collimation system to ensure that potentially destructive halo particles are absorbed before they hit vulnerable elements. Beam-based alignment of the collimators is required to ensure that they are positioned for maximum cleaning efficiency. The alignment procedure relies on feedback from Beam Loss Monitors, and is currently being automated to speed it up. This paper describes a method for automatically selecting a threshold for the beam loss signal during alignment, based on an empirical analysis of collimator alignment data over one year of operation. The results achieved with threshold selection during alignments at $4 \mathrm{TeV}$ are presented.
\end{abstract}

Keywords- Large Hadron Collider; collimators; signal threshold

\section{INTRODUCTION}

The Large Hadron Collider (LHC) at CERN commenced operations in 2008. It is designed to accelerate two proton or heavy ion beams to approach the speed of light, before colliding them in four points where the experimental detectors are located [1]. Each beam is composed of 2808 bunches with $1.15 \times 10^{11}$ particles per bunch. The particles are normally distributed in the transverse plane, and the tails or halo regions are populated by dynamic processes, such as intra-beam scattering and diffusion. The halo particles can damage or irradiate machine components if they are not removed from circulation.

For these reasons, a collimation system consisting of 86 movable collimators is in place to scatter and absorb halo particles, thus protecting the LHC [2]. Each collimator is made up of two blocks or 'jaws' of carbon or tungsten material, whose length may be $0.6 \mathrm{~m}$ or $1.0 \mathrm{~m}$ depending on the collimator type. A photograph of a collimator prior to installation in the LHC is shown in Fig. 1. The collimator jaws need to be positioned symmetrically on either side of the beam and in parallel to the beam direction to be able to remove halo particles with maximum efficiency.

The symmetric jaw positions can be calculated if the beam centers at each collimator location are known. If the jaws are moved in small steps (typically $5 \mu \mathrm{m}$ ) until they

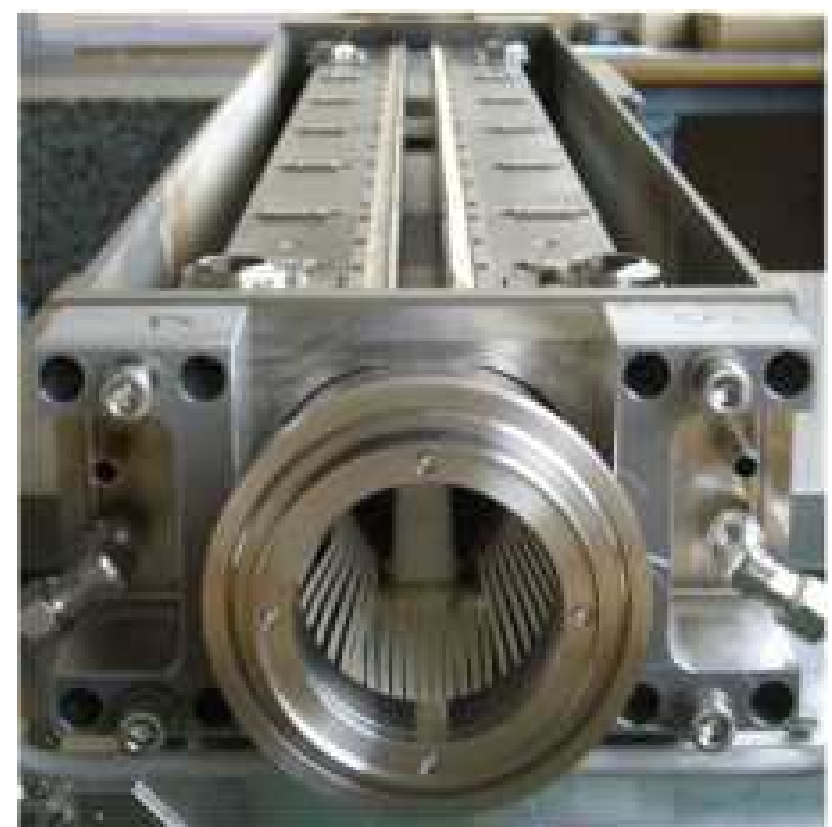

Figure 1. Frontal view of a collimator before installation in the LHC The beam passes through the two blocks of carbon or tungsten material ('jaws'), which are positioned symmetrically around the beam center.

touch the beam halo on both sides, the beam center can be calculated as the average of the two aligned jaw positions. When the jaw touches the beam, a loss spike is observed in the signal of a Beam Loss Monitor (BLM) downstream of the collimator.

As part of efforts to automate and reduce the time required for an alignment of all 86 collimators, a BLM feedback loop was developed, whereby the jaws are moved in automatically until a pre-defined BLM signal threshold is exceeded [3]. The threshold must be set at the right level: if it is too high, the jaw scrapes excessive beam away, and the beam is possibly dumped due to the ensuing high losses. If the threshold is set too low, the jaw stops prematurely and errors are introduced in determining the beam centers.

Approximately 3600 BLM ionization chambers are installed around the $\mathrm{LHC}$ ring to detect ionizing radiation deriving from particle losses [4], [5]. Figure 2(a) shows a 


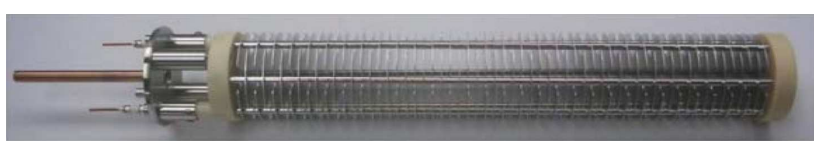

(a) A BLM ionization chamber without its casing

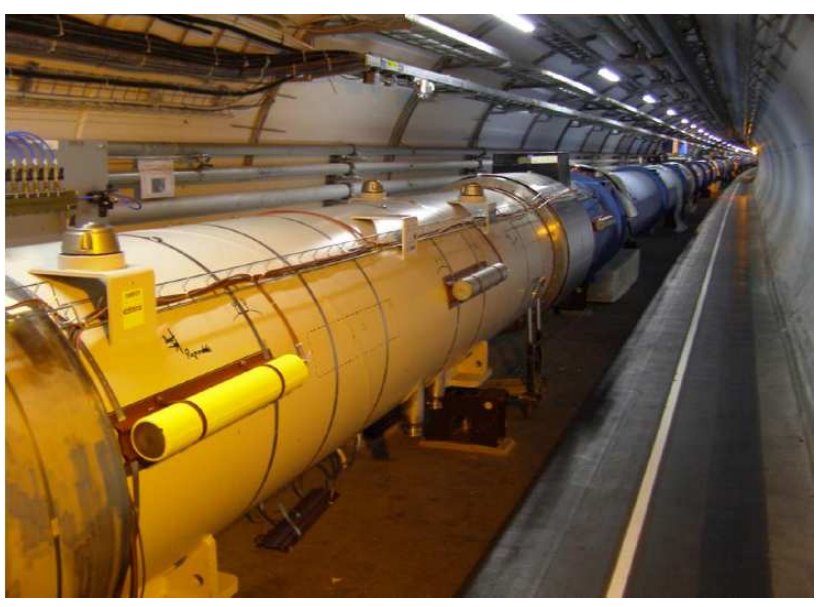

(b) BLMs attached to the walls of the LHC

Figure 2. Beam loss monitor without its casing (top) and installed in the LHC (bottom), from [5].

BLM without its external casing, while an example of a BLM installation in the LHC tunnel is shown in Fig. 2(b). BLMs are also placed within a few meters downstream of the collimators to detect beam losses resulting from halo particles impacting with the collimator jaws.

The ionizing radiation readings, measured in units of Gy, induce a current which is integrated over 12 periods of time [6]. The integration time periods or running sums range from $40 \mu \mathrm{s}$ to $84 \mathrm{~s}$. As the collimators form part of a multiturn and multi-stage cleaning system, the 1.3 s running sum is used to analyze its performance, as it contains information about steady-state losses over hundreds of turns. The BLM data with this running sum can be acquired at a rate of $1 \mathrm{~Hz}$.

In signal processing, amplitude thresholds are set to automatically identify regions of interest from noise. They are often used as a spike detection technique. An example of such a technique is presented in [7], where the threshold is calculated as a multiple of the estimated noise level in the signal. Automatic selection of the BLM signal threshold during collimator alignment would contribute greatly to automating the procedure even further.

This paper is organized as follows. The second section presents the typical BLM signal patterns encountered during LHC collimator alignment. Section III describes the data analysis performed on the BLM signals and thresholds acquired from alignment campaigns held in 2011. The implementation of the thresholding technique in the Java collimator alignment application and results from alignments in 2012 are presented in Section IV.

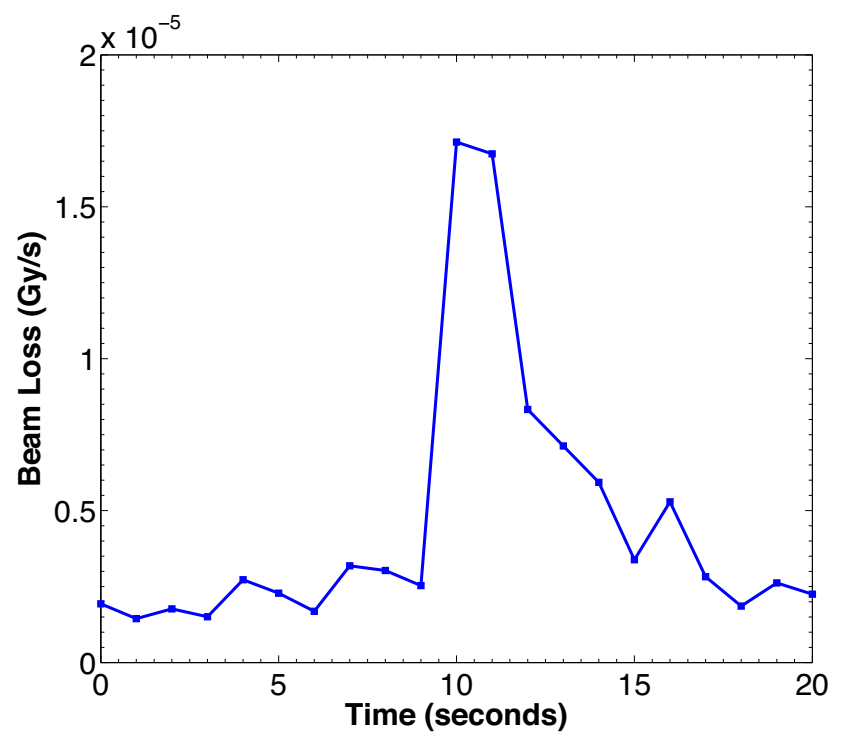

Figure 3. Typical example of a BLM signal spike resulting after an inward collimator jaw movement at $t=9 \mathrm{~s}$.

\section{BLM Signals during COLlimator AlignMent}

When beam is present in the LHC, the BLM signals are generally steady-state, indicating a constant removal of halo particles by the collimators. As soon as a jaw touches the edge of the beam, which normally occurs at $\sim 4 \sigma$, a spike is observed in the BLM signal. The spike amplitude is proportional to the proximity of the jaw to the beam center, and generally ranges from $1 \times 10^{-6} \mathrm{~Gy} / \mathrm{s}$ and $1 \times 10^{-4} \mathrm{~Gy} / \mathrm{s}$. The decay time increases with the beam energy [8], and is typically 3 to 20 seconds.

A typical example of such as spike is provided in Fig. 3. Once the spike has decayed, the steady-state signal is generally higher than it was before the spike. This reflects the fact that the collimator jaw is now closer to the beam center, and is therefore scraping away more halo particles than previously.

\section{DATA ANALYSIS}

In the 2011 LHC run, four major alignment campaigns were carried out using the semi-automatic alignment tool [3] to determine the beam centers at the collimators during different parts of the LHC machine cycle. The alignments are performed at $450 \mathrm{GeV}$ (injection energy), 3.5 TeV flat top, 3.5 TeV after the beam size is reduced (squeezed) in the experimental points and at $3.5 \mathrm{TeV}$ with the beams in collisions. The thresholds at the start of each repetitive jaw movement towards the beam were input manually by the operator. This provides a lot of training data which can be exploited when attempting to find an automatic technique for setting the threshold.

When setting the threshold at time $t=0$, the operator generally gives more importance to the most recent values 
( $B L M_{t=-5}$ to $B L M_{t=0}$ ), but must also consider values up to $B L M_{t=-19}$. This is because a spike occurring previously might still be decaying back to a steady-state value, although a fixed waiting period of $\sim 10$ seconds is enforced before moving the same or another jaw. Mathematically, the assignment of different priorities to the data depending on their occurrence in time can be expressed by means of the exponentially weighted moving average (EWMA), which is given as follows for a 20 second window:

$$
E W M A_{B L M}=\frac{\sum_{i=1}^{20} e^{i} \times B L M_{t=i-20}}{\sum_{i=1}^{20} e^{i}}
$$

A total of 475 samples of the steady-state BLM signal in 20 second windows and the subsequent correct threshold set by the operator (training threshold) were extracted from the logged data. Two examples of BLM signal windows and the corresponding EWMA and threshold are shown in Table I. In the first example, the signal is stable at $\sim 1.00 \times 10^{-6} \mathrm{~Gy} / \mathrm{s}$, while in the second example, the window includes part of the temporal decay of a previous loss spike. The larger weights were assigned to the most recent values.

If the training thresholds are plotted as a function of the EWMA, a power fit can be applied to the data as shown in Fig. 4. The fit is made using the Ezyfit MATLAB tool [9], which uses MATLAB's built-in fminsearch function based on the Nelder-Mead method. There is a correlation coefficient of 0.96611 between the measured data and the fit. The training thresholds are discrete as the operators could select from a drop-down menu with a list of pre-determined thresholds to speed up the alignment [3]. The threshold set at the start of each jaw movement can therefore be calculated as:

$$
S_{i}^{\text {Thres }}=0.53584 \times\left(E W M A_{B L M}\right)^{0.85916}
$$

The maximum threshold that can be set is fixed at $1 \times 10^{-4}$ $\mathrm{Gy} / \mathrm{s}$, which is an order of magnitude below the BLM dump thresholds. As the alignment generally takes place at 3.5 to $4 \sigma$ from the beam center, the steady-state BLM signal ranges from $5 \times 10^{-7}$ to $8 \times 10^{-5}$, and hence the maximum threshold should rarely be reached.

\section{RESUlTS}

The BLM signal threshold selection function in Eq. (2) was implemented into the top-level Java collimator control application [10]. Testing was carried out during alignments held in March 2012. A plot showing the synchronized BLM signal, calculated threshold and collimator jaw gap is given in Fig. 5. The data is taken from an alignment of a primary collimator (TCP) at $4 \mathrm{TeV}$ flat top. The jaw gap is plotted, rather than the individual jaw positions, to give a clearer picture of the small jaw movements. During the tests, no time-consuming beam dumps were triggered due to high losses, which confirms the effectiveness of the loss threshold function.

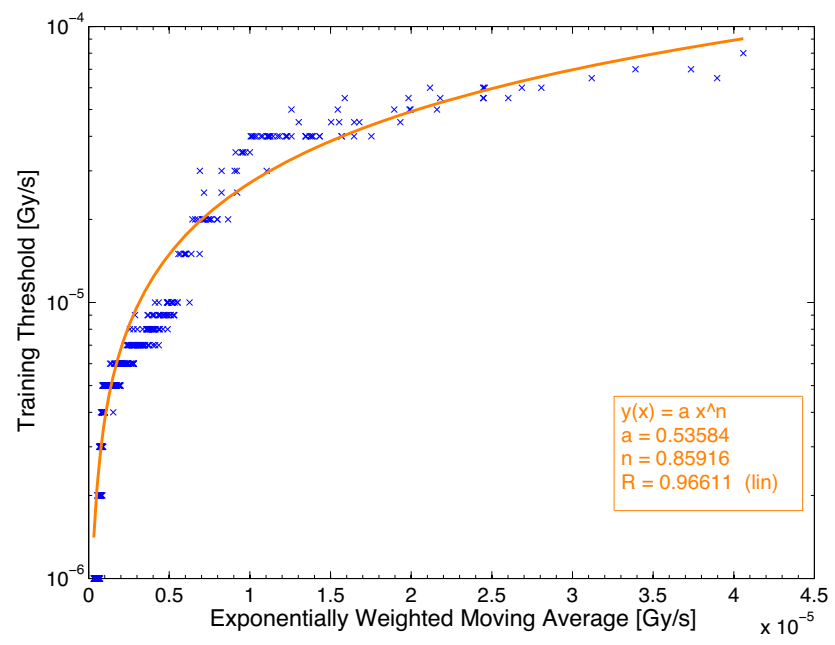

Figure 4. Loss thresholds applied before the start of a jaw movement as a function of the exponentially weighted moving average of the BLM signal. A power fit can be applied to the data.

Table I

TWO EXAMPLES OF BLM SIGNAL WINDOWS, THE CORRESPONDING EWMA, THE THRESHOLD SET BY THE OPERATOR AND THE

\begin{tabular}{|c|c|c|}
\hline$t[s]$ & BLM Value $[\mathrm{Gy} / \mathrm{s}]$ & BLM Value $[\mathrm{Gy} / \mathrm{s}]$ \\
\hline-19 & $1.66 \times 10^{-6}$ & $8.88 \times 10^{-6}$ \\
\hline-18 & $1.51 \times 10^{-6}$ & $8.83 \times 10^{-6}$ \\
\hline-17 & $1.39 \times 10^{-6}$ & $6.80 \times 10^{-6}$ \\
\hline-16 & $1.35 \times 10^{-6}$ & $6.90 \times 10^{-6}$ \\
\hline-15 & $1.31 \times 10^{-6}$ & $6.96 \times 10^{-6}$ \\
\hline-14 & $1.18 \times 10^{-6}$ & $6.50 \times 10^{-6}$ \\
\hline-13 & $1.31 \times 10^{-6}$ & $6.94 \times 10^{-6}$ \\
\hline-12 & $1.27 \times 10^{-6}$ & $6.76 \times 10^{-6}$ \\
\hline-11 & $1.25 \times 10^{-6}$ & $6.53 \times 10^{-6}$ \\
\hline-10 & $1.23 \times 10^{-6}$ & $6.52 \times 10^{-6}$ \\
\hline-9 & $1.19 \times 10^{-6}$ & $5.99 \times 10^{-6}$ \\
\hline-8 & $1.10 \times 10^{-6}$ & $5.50 \times 10^{-6}$ \\
\hline-7 & $1.15 \times 10^{-6}$ & $5.74 \times 10^{-6}$ \\
\hline-6 & $1.31 \times 10^{-6}$ & $5.66 \times 10^{-6}$ \\
\hline-5 & $1.12 \times 10^{-6}$ & $4.86 \times 10^{-6}$ \\
\hline-4 & $1.10 \times 10^{-6}$ & $4.02 \times 10^{-6}$ \\
\hline-3 & $1.14 \times 10^{-6}$ & $4.17 \times 10^{-6}$ \\
\hline-2 & $1.06 \times 10^{-6}$ & $4.12 \times 10^{-6}$ \\
\hline-1 & $1.00 \times 10^{-6}$ & $4.16 \times 10^{-6}$ \\
\hline 0 & $9.72 \times 10^{-7}$ & $3.98 \times 10^{-6}$ \\
\hline EWMA & $9.95 \times 10^{-7}$ & $4.05 \times 10^{-6}$ \\
\hline Training Threshold & $4.00 \times 10^{-6}$ & $1.00 \times 10^{-5}$ \\
\hline Calculated Threshold & $3.73 \times 10^{-6}$ & $1.25 \times 10^{-5}$ \\
\hline
\end{tabular}
CALCULATED THRESHOLD 


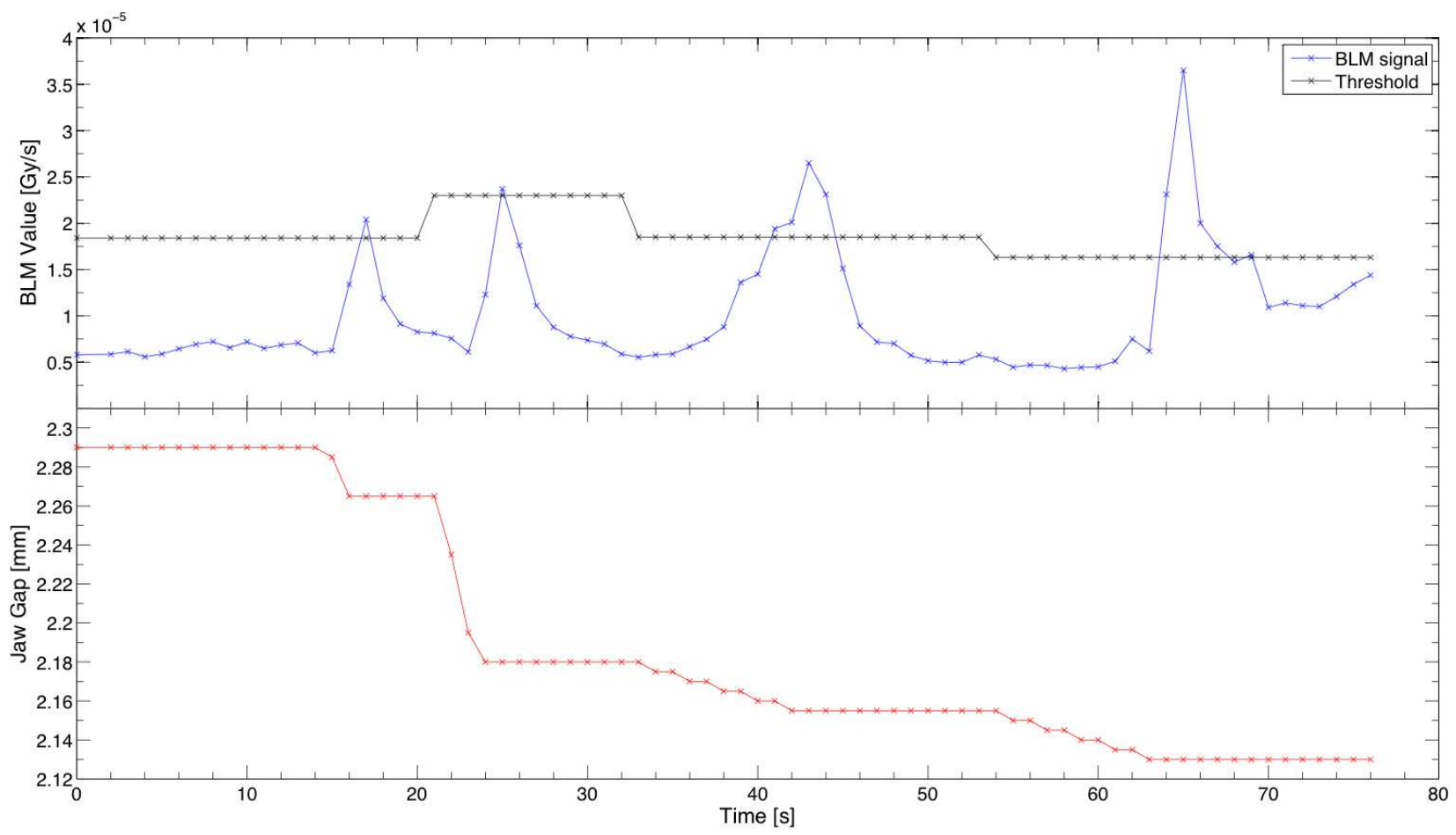

Figure 5. The BLM signal and calculated threshold (top) and the collimator jaw gap (bottom) as a function of time. The individual jaws are aligned four times, and at the start of each jaw movement a new threshold is set depending on the previous BLM values (t[0] $=08: 55: 57,29.03 .2012$ ).

\section{CONCLUSION}

In the LHC, a beam cleaning system composed of collimators removes potentially destructive halo particles before they can reach crucial machine elements, such as the superconducting magnets. The cleaning efficiency depends on the correct positioning of the collimator jaws with respect to the beam. The jaw positions are determined via beam-based alignment, and the software tool used to remotely control the LHC collimators is being improved to automate and speed up the alignment procedure. The alignment relies on feedback from Beam Loss Monitors to determine whether the jaw has touched the beam.

This paper presented a technique for automatically selecting a loss threshold, to ensure that the jaw stops at the precise moment when it touches the beam. The threshold was previously set manually, and hence an abundance of training data is available. A model was developed, based on the exponentially weighted moving average of the BLM data. The model allows the threshold to be calculated at the start of each jaw movement via a simple relation.

\section{ACKNOWLEDGMENT}

This research is funded by EuCARD ColMat WP8 and the University of Malta. The authors would like to thank F. Burkart, M. Cauchi, D. Deboy and B. Salvachua for help during collimator alignments in the CERN Control Center.

\section{REFERENCES}

[1] Report No. CERN-2004-003-V1, edited by O. S. Brüning, P. Collier, P. Lebrun, S. Myers, R. Ostojic, J. Poole, P. Proudlock (2004).

[2] R. W. Aßmann et al. Requirements for the LHC Collimation System. In Proceedings of EPAC'02, Paris, France, 2002, pp. 197-199.

[3] G. Valentino, R. W. Aßmann, R. Bruce, S. Redaelli, A. Rossi, N. Sammut, D. Wollmann. Semiautomatic Beam-Based LHC Collimator Alignment. Phys. Rev. Spec. Top. Accel. Beams 15, 051002 (2012).

[4] B. Dehning et al. The LHC Beam Loss Measurement System. In Proceedings of PAC'07, Albuquerque, USA, 2007, pp. 41924194.

[5] M. Stockner, B. Dehning, C. Fabjan, E. B. Holzer, D. Kramer. Classification of the LHC BLM Ionization Chamber. In Proceedings of DIPAC'07, Venice, Italy, 2007, pp. 328-330.

[6] C. Zamantzas. The Real-Time Data Analysis and Decision System for Particle Flux Detection in the LHC Accelerator at CERN. CERN-THESIS-2006-037.

[7] M. Rizk, P. D. Wolf. Optimizing the Automatic Selection of Spike Detection Thresholds using a Multiple of the Noise Level. Med Biol Eng Comput. 47(9), 955-966, 2009.

[8] G. Valentino, R. W. Aßmann, R. Bruce, F. Burkart, L. Lari, S. Redaelli, A. Rossi, N. Sammut, D. Wollmann. Modeling and Simulation of LHC Beam-Based Collimator Setup. In Proceedings of IPAC'12, New Orleans, USA, 2012, pp. 20592061.

[9] F. Moisy, software available at http://www.fast.u-psud.fr/ezyfit.

[10] S. Redaelli, R. W. Aßmann, M. Jonker, M. Lamont. CERNEDMS Report No. LHC-TCT-ES-0001, 2007. 